\title{
Schulniveau- und Leistungserwartungen von Lehrpersonen und Leistungsentwicklung beim Übergang in die Sekundarstufe I
}

Markus P. Neuenschwander \& Edith Niederbacher

\section{Zusammenfassung}

Lehrpersonenerwartungen beeinflussen Leistungen. Diese Erwartungen können sich auf die Schülerleistungen oder das Schulniveau beziehen, können implizit oder explizit sein. Erwartungseffekte auf die Leistungsentwicklung vom 5. ins 6. sowie vom 5. ins 7. Schuljahr wurden anhand einer Schweizer Stichprobe vergleichend für Schülerinnen und Schüler mit Übertritt in die Sekundarstufe I nach dem 5. Schuljahr $(N=499)$ und mit Übertritt in die Sekundarstufe I nach dem 6. Schuljahr $(N=374)$ regressionsanalytisch überprüft. Die Ergebnisse zeigen, dass implizite, nicht kommunizierte Schulniveauerwartungen von Lehrpersonen durch explizite leistungsbezogene Lehrpersonenerwartungen vermittelt werden. Kommunizierte Schulniveauerwartungen von Primarschullehrpersonen prognostizieren die differenzielle Leistungsentwicklung in Mathematik und Deutsch in den verschiedenen Schulniveaus sehr gut. Verschiedene Wirkungen von Lehrpersonenerwartungen werden diskutiert.

\section{Schlagworte}

Erwartungen, Leistungen, Schulübergang, Pygmalion 


\section{$1 \quad$ Einleitung und Fragestellung}

Immer wieder zeigten Studien und Metaanalysen, dass Lehrpersonenerwartungen die Leistungen von Schülerinnen und Schülern beeinflussen (Rosenthal \& Jacobson, 1974; Dusek \& Joseph, 1983). Lehrpersonenerwartungen tragen zur Erklärung bei, wie Unterricht die Leistungsentwicklung der Kinder beeinflusst (Eccles \& Roeser, 2011). Allerdings richten Lehrpersonen bei gleichen Leistungen der Schülerinnen und Schüler geringere Erwartungen an Kinder aus tiefen sozialen Schichten, mit Migrationshintergrund oder mit wenig familiärer Unterstützung (Tenenbaum \& Ruck, 2007, Jussim, Harber, Crawford, Cain \& Cohen, 2005; Neuenschwander, 2014). Bisher wurden aber die Wirkungen von leistungsbezogenen Lehrpersonenerwartungen nicht mit impliziten, nicht kommunizierten oder expliziten, kommunizierten Schulniveauerwartungen verglichen. Diese Unterscheidung ist hilfreich, weil sich Lehrpersonenerwartungen zwischen Schulfächern (Eccles, Wigfield \& Schiefele, 1998), der Beobachtungseinheit Individuum und Klasse (Friedrich, Flunger, Nagengast, Jonkmann \& Trautwein, 2015) sowie zwischen den Leistungen und Bildungsverläufen (Englund, Egeland, Collins, 2008) unterscheiden. Wie wirken sich verschiedene Arten von Lehrpersonenerwartungen und ihre Wechselwirkungen auf die Leistungsentwicklung von Schülerinnen und Schülern in Mathematik und Deutsch beim Übergang in die Sekundarstufe I aus? Sind Erwartungseffekte auch nach einem Schulübertritt nachweisbar?

Bereits Rosenthal (1968) berichtete von einer Intelligenzsteigerung bei Versuchspersonen durch hohe Erwartungen der Versuchsleitung, was mit Verweis auf die griechische Sage als Pygmalion-Effekt bezeichnet wurde. Im Anschluss daran berichteten Rosenthal \& Jacobs (1974) Effekte von Lehrpersonenerwartungen auf die Intelligenz von Schülerinnen und Schülern. Auch wenn diese Studien teilweise wegen methodischer Mängel kritisiert wurden, wurde im Anschluss daran in vielen Studien und Metaanalysen ein Einfluss von Lehrpersonenerwartungen auf die Schülerleistungen gezeigt (z. B. Dusek \& Joseph, 1983, Überblick in Ludwig, 1998 und Jussim \& Harber, 2005), wenngleich die Effekte oft nur klein bis mittelmässig waren (Jussim, Robustelli \& Cain, 2009). Obwohl Lehrpersonenerwartungen hinsichtlich der Schülerleistungen meist akkurat sind, bilden sie auch selbsterfüllende Prophezeiungen und schaffen somit eine soziale Realität (Jussim, Eccles, Madon, 1996). Lehrpersonenerwartungen tragen dazu bei, dass sich Kinder entsprechend dieser Erwartungen verändern.

Die Lehrpersonenerwartungen an die Schülerinnen und Schüler haben verschiedene Inhalte. Wir unterscheiden leistungsbezogene Erwartungen und Schulniveauerwartungen. Leistungsbezogene Lehrpersonenerwartungen beschreiben die Einschätzung der Schülerleistungen durch die Lehrperson im Klassenvergleich. 
Schulniveauerwartungen unterscheiden wir nach der Form in implizite, nicht kommunizierte und explizite, kommunizierte Schulniveauerwartungen. Schulniveauerwartungen von Lehrpersonen beziehen sich auf eines von drei Schulniveaus der Sekundarstufe I, welchem ein Kind voraussichtlich zugewiesen wird. Sie resultieren aus einer Einschätzung der Schulkarriere des Kindes durch die Lehrperson in der Primarstufe (Ditton \& Krüsken, 2009).

\subsection{Nicht kommunizierte Schulniveauerwartungen und Leistungserwartungen}

Lehrpersonen schätzen das zukünftige Schulniveau der Sekundarstufe I der Schülerinnen und Schüler ihrer Klasse bereits zwei Jahre vor dem Übertritt in die Sekundarstufe I, d.h. vor Beginn der Beurteilungsphase, recht genau ein (Ditton \& Krüsken, 2009). Ihre Einschätzungen sind hierbei zuverlässiger als diejenigen der Eltern (Neuenschwander, Rottermann, Rösselet, Niederbacher, 2015). Diese Schulniveauerwartung basiert auf einer impliziten, d.h. grob eingeschätzten Beurteilung der schulischen Leistungen und der Schülerintelligenz, aber auch auf der familiären Unterstützung und der Erwartungen von Eltern an ihr Kind (Neuenschwander et al., 2015; Glock, Kneer \& Kovacs, 2013). Sie wird nicht explizit kommuniziert. Die nicht kommunizierte Schulniveauerwartung wirkt sich auf die Leistungsentwicklung aus, weil sie mit entsprechenden impliziten Leistungserwartungen an die Kinder verbunden ist bzw. durch diese im Sinn einer Mediation erklärt wird. Wenn Lehrpersonen die Zuweisung in ein Schulniveau mit hohen Anforderungen erwarten, erwarten sie hohe Leistungen, mit denen die hohen Schulniveauanforderungen erfüllt werden können. Konkret: Wenn Lehrpersonen im 5. Schuljahr die Zuweisung in ein Schulniveau mit hohen Anforderungen im 7. Schuljahr erwarten, müssten die Leistungen der Schülerinnen und Schüler vom 5. zum 6. Schuljahr stärker zunehmen als bei einer Erwartung in ein Schulniveau mit tiefen Anforderungen.

Nach Harris \& Rosenthal (1985) beeinflussen Lehrpersonenerwartungen die Schülerleistungen, weil Lehrpersonen bei hohen Erwartungen den Schülerinnen und Schülern anspruchsvollere Aufgaben zuweisen, motivationsförderliche Rückmeldungen geben, eine positivere Beziehung zu ihnen haben und ihnen mehr Interaktionsgelegenheiten bieten. Damit übereinstimmend konnten Rubie-Davis, Peterson, Sibley \& Rosenthal (2015) experimentell Lehrpersonenerwartungen verändern und zeigen, dass sich die Erwartungen über die Interaktionen mit den Kindern auf die Leistungen auswirken. In Weiterführung dazu wird angenommen, dass Kinder die Erwartungen ihrer Lehrpersonen verinnerlichen, sich so 
verhalten, dass sie diesen Erwartungen entsprechen und die Wirkungen auch bei einem Lehrpersonenwechsel vorhanden bleiben. Entsprechend fanden Studien von Brophy (1985) Wirkungen von Lehrpersonenerwartungen auf die Schülerleistungen auch bei einem Lehrpersonenwechsel. Vor diesem Hintergrund wurden folgende Hypothesen formuliert:

Hypothese 1 Nicht kommunizierte Schulniveauerwartungen von Primarschullehrpersonen beeinflussen die Schülerleistungen in Mathematik und Deutsch (1a) bereits am Ende der Primarschule und (1b) nach dem Eintritt in die Sekundarstufe I.

Hypothese 2 Der Einfluss der nicht kommunizierten Schulniveauerwartungen von Primarschullehrpersonen auf Schülerleistungen in Mathematik und Deutsch wird sowohl (2a) am Ende der Primarschule als auch (2b) nach dem Eintritt in die Sekundarstufe I durch leistungsbezogene Lehrpersonenerwartungen vermittelt (Mediation).

\subsection{Kommunizierte Schulniveauerwartungen}

Das von Lehrpersonen schon vor dem Beginn des Selektionsverfahrens für die Sekundarstufe I erwartete Schulniveau wird nur teilweise reflektiert. Es wird den Schülerinnen und Schülern sowie den Eltern in der Regel nicht explizit kommuniziert. Im Unterschied dazu werden die Schulniveauerwartungen von Lehrpersonen am Ende einer Beobachtungs- und Beurteilungsphase mit den Eltern im Rahmen von sogenannten Übertrittsgesprächen gemäss Vorgaben kantonaler Gesetze diskutiert. Je nach Kanton legen Lehrpersonen ein Schulniveau fest, in das ein Kind etwa ein halbes Jahr später übertreten wird und kommunizieren dies dem Kind und dessen Eltern. In anderen Kantonen legen Lehrpersonen und Eltern im Konsensverfahren ein Schulniveau fest. Dieser vorläufige Schulniveauentscheid kann aufgrund von Prüfungsergebnissen oder Rekursen korrigiert werden, ist aber in der Regel endgültig. Der vorläufige Entscheid äussert sich in einer kommunizierten Schulniveauerwartung der Lehrperson, weil sie den Eltern und ihren Kindern, aber auch den anderen Kindern der Klasse explizit bekannt ist. Entsprechend sollte die kommunizierte Schulniveauerwartung von Lehrpersonen mit der Schulniveauerwartung von Eltern deutlich stärker korrespondieren als die nicht kommunizierte Schulniveauerwartung. Daraus resultiert zur Validierung der Interpretation der Schulniveauerwartung die dritte Hypothese: 
Hypothese 3 Kommunizierte Schulniveauerwartungen von Lehrpersonen stimmen mit den Schulniveauerwartungen von Eltern stärker überein als nicht kommunizierte Schulniveauerwartungen (Validierungshypothese).

Analog zu den nicht kommunizierten Schulniveauerwartungen führen auch kommunizierte hohe Schulniveauerwartungen von Lehrpersonen zu einer Leistungszunahme der Schülerinnen und Schüler. Wir nehmen an, dass während die nicht kommunizierten Schulniveauerwartungen zu einer beiläufigen, nicht reflektierten Förderung von Kindern führen, die kommunizierten Schulniveauerwartungen mit einer geplanten inneren Differenzierung im Unterricht einhergehen. Analog zur differenziellen Förderung in unterschiedlich anspruchsvollen Schulniveaus mit entsprechend unterschiedlich starken Leistungszuwächsen (vgl. Schereneffekte nach Baumert, Stanat \& Waterman, 2006) dürfte die erwartete Zuweisung in ein Schulniveau mit hohen Anforderungen zu einer angereicherten Förderung der Kinder, ergänzt mit einem entsprechenden Fähigkeitsselbstkonzept und Leistungsmotivation führen. Diese angereicherte Förderung setzt sich nach Eintritt in ein Schulniveau mit hohen Anforderungen fort, verstärkt durch entsprechende Kompositionseffekte (Baumert et al., 2006). Demnach wirken sich kommunizierte Schulniveauerwartungen nicht aufgrund von Leistungserwartungen der Lehrpersonen auf die Leistungen aus, sondern aufgrund der geplanten, an das Kind angepassten, aber ausgeprägten Förderung. Folgende Hypothesen werden daher überprüft:

Hypothese 4 Kommunizierte Schulniveauerwartungen von Primarschullehrpersonen beeinflussen die Leistungsentwicklung in Mathematik und Deutsch (4a) beim Übergang in die Sekundarstufe I und (4b) nach dem Eintritt in die Sekundarstufe I.

Hypothese 5 Der Einfluss kommunizierter Schulniveauerwartungen von Lehrpersonen auf Schülerleistungen in Mathematik und Deutsch wird (5a) weder beim Verbleib in der Primarschule (6. Schuljahr) noch (5b) beim Übergang in die Sekundarstufe I (7. Schuljahr) durch leistungsbezogene Lehrpersonenerwartungen vermittelt (fehlende Mediation).

\subsection{Fachunspezifität von Erwartungseffekten}

Zur Fachspezifität von Erwartungseffekten gibt es kaum Forschungsliteratur. Die Prozesse, die der Wirkung von Erwartungseffekten zu Grunde liegen, sind unab- 
hängig vom Fachinhalt (Eccles et al., 1983). Entsprechend wurden Erwartungseffekte auf die Leistungen in verschiedenen Schulfächern gezeigt (Mathematik: z. B. Friedrich, et al., 2015, Naturwissenschaften: z. B. Trouilloud, Sarrazin, Martinek, Guillet, 2002; Lesen: McKown \& Weinstein, 2008). Die Wirkungen der leistungsbezogenen Erwartungen sowie der nicht kommunizierten und kommunizierten Schulniveauerwartungen von Lehrpersonen werden daher in Bezug auf zwei Schulfächer, Mathematik und Deutsch, geprüft.

\section{$2 \quad$ Methode}

\subsection{Stichprobe}

Die Hypothesen wurden mit Daten aus der Schweizer Studie „Wirkungen der Selektion WiSel“ überprüft. Das Design der Studie basiert auf drei Messzeitpunkten (5., 6. und 7. Schuljahr), wobei sich die beiden Vergleichsgruppen im Zeitpunkt des Übertritts in die Sekundarstufe I unterschieden: In den Kantonen Aargau und Basel-Landschaft traten die Schülerinnen und Schüler nach dem 5. Schuljahr in die Sekundarstufe I über, in den Kantonen Bern und Luzern nach dem 6. Schuljahr. Zum dritten Messzeitpunkt befand sich die Gruppe mit frühem Übertritt bereits ein Jahr in der leistungsdifferenzierten Sekundarstufe I (mit drei Schulniveaus: Grundanforderungen, erweiterte Anforderungen, hohe Anforderungen), während die Gruppe mit spätem Übertritt diesen gerade erst vollzogen hatte. Aus den vier Kantonen wurden mit dem Einverständnis der kantonalen Verwaltungen zufällig Schulen ausgewählt und deren Schulleitungen und Klassenlehrpersonen um das Einverständnis zur Teilnahme an der Studie gebeten. Die Eltern meldeten ihre Kinder schriftlich zur Teilnahme an der Studie an. Die Stichprobe ist für die involvierten Kantone repräsentativ.

Bei der Gruppe mit frühem Übertritt unterschied sich die Zusammensetzung der Längsschnittstichprobe (Teilnahme im 5. und 6. Schuljahr) nicht systematisch von der restlichen Stichprobe (Leistungen Mathematik t1: $t=1.07, d f=652, n s, d=0.09$, Leistungen Deutsch t1: $t=0.83, d f=646, n s, d=0.07$, Leistungsbezogene Lehrpersonenerwartungen $\mathrm{t} 1$ : $t=0.18, d f=635, n s, d=0.015$, Schulniveauerwartungen von Lehrpersonen t1: $\left.C h i^{2}=1.354, d f=2, n s\right)$. Bei der Gruppe mit spätem Übertritt gab es zwischen Längsschnitt- und restlicher Stichprobe gewisse signifikante Unterschiede, diese sind jedoch aufgrund der geringen Effektstärken vernachlässigbar (Leistungen Mathematik t1: $t=2.99, d f=838, p<0.05, d=0.26$, Leistungen Deutsch t1: $t=4.46, d f=837, p<0.05, d=0.40$, Leistungsbezogene Lehrpersonenerwartun- 
gen: $t=3.52, d f=709, p<0.05, d=0.34$, Schulniveauerwartungen von Lehrpersonen: $\left.\mathrm{Chi}^{2}=7.63, d f=2, n s\right)$. Die Befunde zu den Rücklaufverzerrungen vom 6. zum 7 . Schuljahr waren ähnlich.

Da keine bzw. vernachlässigbare Rücklaufverzerrungen zwischen der Querschnittstichprobe zu t1 und der Längsschnittstichprobe vorlagen, wurden fehlende Werte imputiert. Imputiert wurde für die Variablen Leistungstestwerte in Mathematik und Deutsch zu allen drei Messzeitpunkten, IQ, Schulniveauerwartung sowie leistungsbezogene Erwartung der Lehrperson. Kontrolliert wurden die Variablen familiärer sozioökonomischer Status (ISEI), Geschlecht der Schülerinnen und Schüler und die Schulniveauzugehörigkeit in der 7. Klasse.

Die Längsschnittstichprobe mit imputierten Daten setzt sich wie folgt zusammen: Die Stichprobe der Gruppe mit frühem Übertritt bestand zum ersten Messzeitpunkt aus insgesamt 499 Kindern aus 74 Klassen in 25 Schulen (Anteil Mädchen $=51.3 \%$, Durchschnittsalter 10.9 Jahre). In der Gruppe mit spätem Übertritt befanden sich 374 Kinder aus 37 Klassen in 21 Schulen (Anteil Mädchen $=46.5 \%$, Durchschnittsalter 10.7 Jahre). Weiter liegen Daten aus Fragebogen von 873 Elternteilen und 111 Lehrpersonen vor.

\subsection{Instrumente}

\subsubsection{Leistungstests}

Die Leistungstests in den Promotionsfächern Mathematik und Deutsch bestanden aus einer Auswahl von Aufgaben von Moser, Buff, Angelone und Hollenweger (2011). Zur Auswertung der Tests wurden die Items der jeweiligen Aufgaben dichotom kodiert (falsch: 0 Punkte, richtig: 1 Punkt).

In Tabelle 1 sind die Inhalte und Reliabilitätswerte der Leistungstests in Mathematik und Deutsch zusammenfassend dargestellt. Die Berechnungen beruhen jeweils auf der Gesamtstichprobe des jeweiligen Messzeitpunkts. Die Split-half-Reliabilitäten der Leistungstests sind für beide Fächer und alle Messzeitpunkte zufriedenstellend.

In den Tests beider Fächer wurde in einem Ankeritem-Design eine Teilmenge der Items des früheren Messzeitpunkts zum jeweils folgenden Messzeitpunkt vorgelegt. Basierend auf der Item Response Theorie (IRT; Yen \& Fitzpatrick, 2006) wurde mit der Software R die Linking-Methode von Haberman (2009) angewendet, mit welcher die Schülerleistungen auf einer Metrik abgebildet werden, wodurch die tatsächliche Veränderung der Kompetenzwerte über die drei Messzeitpunkte beschrieben werden kann. Als Kompetenzwerte der Schülerinnen und Schüler wurden sogenannte Weighted-likelihood-Werte (Warm, 1989) ermittelt (Mittelwerte: t1: Mathematik $M=.03, S D=1.09$, Deutsch $M=.04, S D=1.04$; t2: Mathematik 
Tab. 1 Inhalte und Reliabilitätswerte der Leistungstests in Mathematik und Deutsch

\begin{tabular}{|c|c|c|c|}
\hline & \multirow[b]{2}{*}{ Inhalt } & \multicolumn{2}{|c|}{ Split-half-Reliabiliäten } \\
\hline & & $\begin{array}{l}\text { erste Testhälfte vs. } \\
\text { zweite Testhälfte }\end{array}$ & $\begin{array}{c}\text { gerade vs. } \\
\text { ungerade Items }\end{array}$ \\
\hline \multicolumn{4}{|c|}{ Leistungstests Mathematik } \\
\hline Welle 1 & 7 Aufgaben, 39 Items & $r_{\mathrm{SH}}=.80$ & $r_{\mathrm{SH}}=.87$ \\
\hline Welle 2 & 10 Aufgaben, 45 Items & $r_{\mathrm{SH}}=.82$ & $r_{\mathrm{SH}}=.91$ \\
\hline Welle 3 & 8 Aufgaben, 55 Items & $r_{\mathrm{SH}}=.77$ & $r_{\mathrm{SH}}=.91$ \\
\hline \multicolumn{4}{|c|}{ Leistungstests Deutsch } \\
\hline Welle 1 & 6 Aufgaben, 83 Items & $r_{\mathrm{SH}}=.80$ & $r_{\mathrm{SH}}=.92$ \\
\hline Welle 2 & 10 Aufgaben, 85 Items & $r_{\mathrm{SH}}=.82$ & $r_{\mathrm{SH}}=.95$ \\
\hline Welle 3 & 11 Aufgaben, 97 Items & $r_{\mathrm{SH}}=.85$ & $r_{\mathrm{SH}}=.95$ \\
\hline
\end{tabular}

$M=.51, S D=.99 ;$ Deutsch $M=.25, S D=1.13$; 3 : Mathematik: $M=.60, S D=.96$; Deutsch $M=.52, S D=1.18)$.

\subsubsection{Schülerbefragung}

Geschlecht: Das Geschlecht der Schülerinnen und Schüler wurde mit der Angabe „männlich/weiblich“ erhoben.

Schulniveau 7. Klasse: In der 7. Klasse wurde das Schulniveau, in welchem sich die Schülerinnen und Schüler aktuell befanden, erhoben. Falls diese Angabe nicht vorlag, wurde die Information mit der Information der Lehrperson ergänzt. Die Lehrpersonen gaben jeweils am Ende der Primarschule Auskunft, für welche Schulniveaus die Schülerinnen und Schüler angemeldet wurden.

Intelligenz: Die Erfassung der Intelligenz erfolgte mittels der Kurzversion des sprachund kulturunabhängigen Intelligenztests CFT-20-R (1. Testteil; Weiss, 2008). Im Durchschnitt wurden in der Längsschnittstichprobe 36.2 Aufgaben der 56 Aufgaben von den Schülerinnen und Schülern korrekt gelöst. Die individuellen Testwerte wurden an der Normstichprobe der 5. Klasse standardisiert $(M=100, S D=15)$.

\subsubsection{Lehrpersonenbefragung}

Schulniveauerwartung: Im Lehrpersonenfragebogen der ersten Welle gaben die Klassenlehrpersonen für jedes Kind ihrer Klasse an, für welches Schulniveau der Sekundarstufe I sie es empfehlen würden, wenn der Übertritt unmittelbar bevorstünde. Die Antwortmöglichkeiten entsprachen den kantonsspezifischen 
Bezeichnungen und beinhalteten drei bzw. vier mögliche Schulniveaus. Für die hier geplanten Auswertungen wurde die Schulniveauerwartung zuerst in einer dreistufigen Variable abgebildet und nachher in zwei Dummy-Variablen kodiert: Schulniveau mit hohen Anforderungen vs. Schulniveau mit erweiterten und Grundanforderungen sowie Schulniveau mit Grundanforderungen vs. Schulniveau mit erweiterten und hohen Anforderungen.

Im vorliegenden Artikel werden die in der Welle 1 erhobenen Schulniveauerwartungen der Lehrperson für die Gruppe mit spätem Übertritt als nicht kommunizierte Schulniveauerwartungen bezeichnet, da sie zum Erhebungszeitpunkt den Schülerinnen und Schülern sowie deren Eltern noch nicht kommuniziert wurden. Die Schulniveauerwartungen der Lehrperson für die Gruppe mit frühem Übertritt gelten als kommunizierte Schulniveauerwartungen, da das Übertrittsverfahren bereits abgeschlossen war und die Einteilung in das zukünftige Schulniveau mit den Schülerinnen und Schülern sowie den Eltern zum Erhebungszeitpunkt bereits besprochen worden war.

Leistungsbezogene Erwartungen: Die Lehrpersonen der ersten Welle schätzten die Leistungen der Schülerinnen und Schüler in ihren Klassen ein. Gefragt wurde auf einer dreistufigen Skala nach der erbrachten Leistung (unterdurchschnittlich ,1', durchschnittlich ,2' und überdurchschnittlich ,3') in den drei Hauptfächern Mathematik, Deutsch und je nach Kanton Französisch oder Natur-Mensch-Mitwelt im Vergleich zu den übrigen Schülerinnen und Schülern der Klasse $(M=2.09$, $S D=.73, N=873)$.

\subsubsection{Elternbefragung}

Sozioökonomischer Status: Der sozioökonomische Status (ISEI) der Familie wurde anhand der Berufe der ersten und der zweiten Bezugspersonen (Erziehungsberechtigte) zum ersten Messzeitpunkt ermittelt. Die Berufe wurden gemäss der International Standard Occupation Classification (ISCO-08) kodiert. Anschliessend wurde jedem Beruf ein Wert basierend auf dem Standard International Socio-Economic Index of Occupational Status (ISEI-08) zugewiesen (Ganzeboom, 2010). Der jeweils höhere Wert der beiden Bezugspersonen wurde verwendet.

Schulniveauerwartung: Die Eltern wurden zum ersten Messzeitpunkt befragt, welches Schulniveau der Sekundarstufe I ihr Kind nach ihrer Meinung besuchen wird. Kantonsübergreifend erwarteten $13.2 \%$ der Eltern, ihr Kind werde das Niveau mit Grundansprüchen besuchen, $47.1 \%$ erwarteten einen Übertritt in das Niveau mit erweiterten Ansprüchen und 39.7\% der Eltern gaben an, ihr Kind werde voraussichtlich in das Niveau mit hohen Ansprüchen übertreten. 


\subsubsection{Durchführung}

Die schriftlichen Befragungen der Schülerinnen und Schüler, Eltern und Klassenlehrpersonen fanden in den Herbstsemestern der Schuljahre 2011/12, 2012/13 und 2013/14 statt. Die Schülerbefragung und die Leistungstests, die terminlich rund drei Wochen auseinanderlagen, wurden unter Anleitung von geschulten Projektmitarbeitenden im Klassenverband während der Unterrichtszeit durchgeführt. Im Zeitraum zwischen den beiden Klassenbesuchen füllten die Eltern und Lehrpersonen jeweils individuell ihre Fragebogen schriftlich aus.

\section{$3 \quad$ Ergebnisse}

\subsection{Deskriptive Ergebnisse}

Zur Beschreibung der Zusammenhangsmuster der postulierten Prädiktoren wurden getrennt für die zwei Gruppen „früher Übergang“ und „später Übergang“ im nächsten Schritt die Korrelationen berechnet (Tabelle 2). Alle Korrelationen sind signifikant und zum Teil substanziell. Sie stimmen zwischen den zwei Gruppen in hohem Mass überein. Die Schulniveauerwartungen und die Leistungserwartungen von Lehrpersonen korrelierten mit den Leistungen in Mathematik und Deutsch mehrheitlich höher als mit der Intelligenz.

Tab. 2 Pearson-Korrelationen

\begin{tabular}{|c|c|c|c|c|c|c|}
\hline & 1 & 2 & 3 & 4 & 5 & 6 \\
\hline 1 Leistung M 5. Sj & 1 & $.63^{\star * *}$ & $.39^{* * *}$ & $.32^{\star \star \star}$ & $-.52^{\star * \star}$ & $.63^{* * *}$ \\
\hline 2 Leistung D 5. Sj & $.49^{\star * *}$ & 1 & $.34^{* * *}$ & $.33^{\star * *}$ & $-.54^{\star * \star}$ & $.62^{* * *}$ \\
\hline 3 Schülerintelligenz & $.27^{\star * *}$ & $.39^{* * *}$ & 1 & $.22^{\star * *}$ & $-.28^{\star * *}$ & $.31^{\star * *}$ \\
\hline 4 Ni-erwart Lp hoch & $.14^{\star * *}$ & $.35^{\star * *}$ & $.09^{*}$ & 1 & $-.33^{* * *}$ & $.55^{\star * *}$ \\
\hline $5 \mathrm{Ni}$-erwart Lp tief & $-.17^{\star * *}$ & $-.39^{* * *}$ & $-.27^{\star * *}$ & $-.10^{*}$ & 1 & $-.68^{\star * *}$ \\
\hline 6 Leistungserwart & $.32^{\star * *}$ & $.51^{\star * \star}$ & $.24^{\star * *}$ & $.58^{\star \star *}$ & $-.62^{\star * \star}$ & 1 \\
\hline
\end{tabular}

Legende: ${ }^{* * *}: p<.001,{ }^{* *}: p<.01,{ }^{\star}: p<.05 ; \mathrm{M}$ : Mathematik; D: Deutsch; Ni-Erwart Lp hoch: von Lehrpersonen erwartete Zuweisung in ein Schulniveau mit hohen Anforderungen; Ni-Erwart Lp tief: von Lehrpersonen erwartete Zuweisung in ein Schulniveau mit Grundanforderungen; Leistungserwart: leistungsbezogene Lehrpersonenerwartungen oberhalb Diagonale: Gruppe mit frühem Übergang $(N=499)$, unterhalb Diagonale: Gruppe mit spätem Übergang $(N=374)$ 


\subsection{Effekte nicht kommunizierter Schulniveauerwartungen}

Zur Überprüfung der Hypothese 1 wurden getrennt für Mathematik und Deutsch stufenweise Regressionsanalysen mit der Stichprobe der Schülerinnen und Schüler mit spätem Übertritt gerechnet. Mit dieser Teilstichprobe liessen sich Effekte von impliziten, nicht kommunizierten Lehrpersonenerwartungen testen (vgl. Operationalisierung im Methodenteil). Es wurden keine Mehrebenenanalysen gerechnet, weil die Klassenzugehörigkeiten beim Übertritt in die Sekundarstufe I wechselten und um immer die gleichen Analysestrategien zu verwenden, damit die Ergebnisse direkt vergleichbar sind. Die Ergebnisse in Tabelle 3 zeigen, dass in Übereinstimmung mit Hypothese la nicht kommunizierte Schulniveauerwartungen von Lehrpersonen für ein Schulniveau mit hohen Anforderungen, die Leistungsveränderung in Mathematik vom 5. zum 6. Schuljahr der Primarschule erklären $\left(R^{2}\right.$ change $\left.=3.0 \%\right)$, auch nach Kontrolle der Intelligenz und des sozioökonomischen Status der Schülerin/des Schülers. Bezieht man die leistungsbezogenen Lehrpersonenerwartungen im Modell mit ein, erklären diese die Leistungsveränderung signifikant $\left(R^{2}\right.$ change $\left.=1.2 \%\right)$. Mit dem Bootstrappingverfahren (Preacher \& Hayes, 2008, 1000 Bootstrapping-Stichproben) wurde die Mediation der dummy-kodierten Variable "Schulniveauerwartung hohe Anforderungen“ von Lehrpersonen (Referenzkategorien Schulniveau mit erweiterten und tiefen Anforderungen) bzw. Schulniveauerwartung tief (mit Referenzkategorien Schulniveau mit erweiterten und hohen Anforderungen) durch die leistungsbezogenen Lehrpersonenerwartungen auf die Leistungsveränderung auf ihre Signifikanz geprüft. Sowohl die Erwartung auf ein Schulniveau mit hohen Anforderungen (Bootstrapintervall .06 bis .22) als auch die Erwartung auf ein Schulniveau mit Grundanforderungen (Bootstrapintervall -.30 bis -.07) wurden gemäss Hypothese 2 a durch die leistungsbezogenen Lehrpersonenerwartungen teilweise aber signifikant (5\%-Niveau) vermittelt. 
Tab. 3 Regressionsanalysen: Nicht kommunizierte Lehrpersonenerwartungen und Schülerleistungen in Mathematik: Gruppe später Übertritt

\begin{tabular}{|c|c|c|c|c|c|c|}
\hline \multirow[b]{2}{*}{ Modell } & \multicolumn{3}{|c|}{ Leistungen M 6. Sj } & \multicolumn{3}{|c|}{ Leistungen $\mathrm{M}$ 7. Sj } \\
\hline & M1 & M2 & M3 & M1 & M2 & M3 \\
\hline Leistungen M 5. Sj & $.61^{\star * *}$ & $.53^{\star * *}$ & $.47^{\star \star *}$ & $.50^{\star \star *}$ & $.41^{\star \star \star *}$ & $.39^{* * *}$ \\
\hline IQ $5 . \mathrm{Sj}$ & $.10^{*}$ & .07 & .07 & $.21^{* * *}$ & $.18^{\star * *}$ & $.18^{\star \star \star}$ \\
\hline $\begin{array}{l}\text { Sozioökonomischer } \\
\text { Status (ISEI) }\end{array}$ & $.11^{* *}$ & $.08^{*}$ & .08 & $.10^{*}$ & .07 & .07 \\
\hline Schulni Lp hoch 5. Sj & & $.17^{\star \star \star}$ & $.11^{\star}$ & & $.14^{\star \star *}$ & $.12^{\star \star}$ \\
\hline Schulni Lp tief $5 . \mathrm{Sj}$ & & -.08 & .00 & & $-.14^{\star \star}$ & $-.11^{\star}$ \\
\hline L-Erw Lp 5. Sj & & & $.19^{\star \star}$ & & & .07 \\
\hline$F, p$ & $113.5^{\star * *}$ & $77.2^{\star \star *}$ & $67.4^{\star * *}$ & $92.0^{* * *}$ & $63.6^{* * *}$ & $53.2^{* * *}$ \\
\hline$d f_{1} d f_{2}$ & 3,370 & 5,368 & 6,367 & 3,370 & 5,368 & 6,367 \\
\hline$R^{2}$ adj & $47.5 \%$ & $50.5 \%$ & $51.7 \%$ & $42.3 \%$ & $45.6 \%$ & $45.6 \%$ \\
\hline
\end{tabular}

Legende: M: Mathematik; Schulni Lp: Schulniveauerwartungen Lehrperson; L-Erw Lp: Leistungserwartungen von Lehrpersonen; Sj: Schuljahr; ${ }^{* *}: p<.001 ;{ }^{* *}: p<.01 ;{ }^{*}: p<.05$

Im nächsten Schritt wurde für die gleiche Stichprobe die Leistungsveränderung vom 5. ins 7. Schuljahr der Sekundarstufe I durch die gleichen Prädiktoren erklärt. Die Schulniveauerwartungen wurden gemäss Hypothese la signifikant $\left(R^{2}\right.$ change $=0.8 \%$ ). Anders als bei der Prädiktion der Leistungsentwicklung vom 5. zum 6 . Schuljahr wurden die leistungsbezogenen Lehrpersonenerwartungen nun nicht signifikant. Entgegen Hypothese 2 werden die Schulniveauerwartungen hoch (Bootstrapintervall -.03 bis .13) und Schulniveauerwartungen tief (Bootstrapintervall -.19 bis .04) demnach nicht durch Leistungserwartungen mediiert.

Um die Ergebnisse für ein weiteres Schulfach zu replizieren, wurden die gleichen Analysen für das Fach Deutsch wiederholt. Die Ergebnisse (Tabelle 4) sind auf der Konzeptebene ähnlich, auch wenn sich die einzelnen dummy-kodierten Variablen zur Schulniveauerwartung je nach Analyse in ihrer Signifikanz unterscheiden. Hohe Schulniveauerwartungen erklärten die Leistungsveränderung vom 5. ins 6. Schuljahr in Deutsch signifikant $\left(R^{2}\right.$ change $=0.9 \%$ ). Sie werden durch die leistungsbezogenen Lehrpersonenerwartungen signifikant mediiert (Bootstrapintervall .14 bis .33). Tiefe Schulniveauerwartungen erklärten die Leistungsveränderung vom 5. ins 6. Schuljahr hingegen nicht signifikant. In Bezug auf die Leistungsveränderung vom 5. zum 7. Schuljahr zeigte sich das folgende Bild: Tiefe Schulniveauerwartungen erklärten die Leistungsveränderungen vom 5. ins 7. Schuljahr der Sekundarstufe I $\left(R^{2}\right.$ change $=1.2 \%$ ). Sie wurden im Unterschied zum Fach Mathematik in Übereinstim- 
mung mit Hypothese $2 \mathrm{~b}$ durch die leistungsbezogenen Lehrpersonenerwartungen signifikant mediiert (Bootstrapintervall -.39 bis -.12).

Tab. 4 Nicht kommunizierte Lehrpersonenerwartungen und Schülerleistungen in Deutsch: Gruppe später Übertritt (Regressionsanalysen)

\begin{tabular}{|c|c|c|c|c|c|c|}
\hline \multirow[b]{2}{*}{ Modell } & \multicolumn{3}{|c|}{ Leistungen D 6. Sj } & \multicolumn{3}{|c|}{ Leistungen D 7. Sj } \\
\hline & M1 & M2 & M3 & M1 & M2 & M3 \\
\hline Leistungen D 5. Sj & $.65^{\star * *}$ & $.60^{\star * \star}$ & $.52^{\star * \star}$ & $.65^{\star \star \star}$ & $.57^{\star \star *}$ & $.52^{\star * *}$ \\
\hline IQ $5 . \mathrm{Sj}$ & .07 & .05 & .04 & $.14^{\star * *}$ & $.12^{\star *}$ & $.11^{\star \star}$ \\
\hline $\begin{array}{l}\text { Sozioökonomischer } \\
\text { Status (ISEI) }\end{array}$ & $.10^{*}$ & $.08^{*}$ & .07 & .01 & -.01 & -.02 \\
\hline Schulni Lp hoch. Sj & & $.10^{*}$ & .01 & & .04 & -.03 \\
\hline Schulni Lp tief $5 . \mathrm{Sj}$ & & -.05 & .06 & & $-.14^{\star \star}$ & -.05 \\
\hline L-Erw Lp 5. Sj & & & $.28^{\star * \star}$ & & & $.22^{\star \star \star}$ \\
\hline$F, p$ & $122.3^{\star * *}$ & $76.3^{\star * *}$ & $70.9^{\star * *}$ & $123.8^{\star * *}$ & $78.4^{\star * \star}$ & $69.7^{\star * *}$ \\
\hline$d f_{1} d f_{2}$ & 3,370 & 5,368 & 6,367 & 3,370 & 5,368 & 6,367 \\
\hline$R^{2} a d j$ & $49.4 \%$ & $50.2 \%$ & $52.9 \%$ & $49.7 \%$ & $50.9 \%$ & $52.5 \%$ \\
\hline
\end{tabular}

Legende: D: Deutsch; Schulni Lp: Schulniveauerwartungen Lehrperson; L-Erw Lp: Leistungserwartungen von Lehrpersonen; $\mathrm{Sj}$ : Schuljahr; ${ }^{* *}: p<.001 ;{ }^{* *}: p<.01 ;{ }^{*}: p<.05$

Die Ergebnisse zeigen mehrheitlich übereinstimmend für Mathematik und Deutsch signifikante Effekte der impliziten, nicht kommunizierten Schulniveauerwartungen, vor allem bei der Leistungsentwicklung vom 5. zum 6. Schuljahr der Primarschule, etwas schwächer vom 5. zum 7. Schuljahr beim späten Übertritt in die Sekundarstufe I. Der Effekt dieser Schulniveauerwartungen wird durch leistungsbezogene Erwartungen von Lehrpersonen mehrheitlich mediiert, mit Ausnahme der Leistungsentwicklung vom 5. zum 7. Schuljahr in Mathematik.

\subsection{Effekte kommunizierter Schulniveauerwartungen}

Vorbereitende deskriptive Analysen mit der Stichprobe mit frühem Übertritt zeigen, dass die kommunizierte Schulniveauerwartung von Lehrpersonen im 5. Schuljahr und das tatsächliche Schulniveau im 6. Schuljahr in $92.0 \%$ der Fälle übereinstimmt, während die Schulniveauerwartung von Eltern dies nur in 89.3 \% der Fälle mit der Zuweisung tut. In der Stichprobe der Jugendlichen mit spätem Übertritt korrespondiert die Schulniveauerwartung der Lehrperson im 5. Schuljahr in $73.5 \%$ der 
Fälle mit dem Schulniveau im 7. Schuljahr, während die Schulniveauerwartung der Eltern in $69.3 \%$ der Fälle übereinstimmt.

Um die Validität der kommunizierten Schulniveauerwartung von Lehrpersonen zu überprüfen, wurden die Übereinstimmungen zwischen der Schulniveauerwartung der Lehrpersonen und Eltern zwischen den beiden Gruppen verglichen. Die Übereinstimmung ist gemäss Hypothese 3 mit $89.8 \%$ in der Gruppe früher Übertritt signifikant höher als in der Gruppe später Übertritt mit 64.1\% $\left(\mathrm{Chi}^{2}=38.8, d f=1\right.$, $p<.001)$. Schulniveauerwartungen von Lehrpersonen in der Gruppe früher Übertritt sind mit den Eltern mehrheitlich abgestimmt worden und kommuniziert. Damit wird die Operationalisierung gestützt, dass Schulniveauerwartungen je nach Stand des Übertrittsverfahrens unterschiedliche Bedeutungen haben.

Zur Prüfung der Hypothesen 4 und 5 wurde auf die Teilstichprobe der Kinder mit frühem Übertritt zurückgegriffen. In dieser Übertrittssituation korrespondieren die Schulniveauerwartungen mit den kommunizierten Schulniveauerwartungen von Lehrpersonen. Es wurden wiederum stufenweise Regressionsanalysen unter Kontrolle der Schülerintelligenz und des sozioökonomischen Status getrennt für Mathematik und Deutsch gerechnet. Gemäss Tabelle 5 und in Übereinstimmung mit Hypothese 4a erklärten kommunizierte Schulniveauerwartungen von Lehrpersonen in Bezug auf die Zuweisung in ein Schulniveau mit Grundansprüchen die Leistungsentwicklung in Mathematik beim Übergang vom 5. Schuljahr der Primarschule ins 6. Schuljahr der Sekundarstufe I signifikant und substanziell $\left(R^{2}\right.$ change $=10.6 \%$ ). Sowohl die Schulniveauerwartungen hoch (Bootstrapintervall -.13 bis .10) als auch die Schulniveauerwartungen tief (Bootstrapintervall -.10 bis .13) wurden gemäss Hypothese 5 a nicht durch leistungsbezogene Lehrpersonenerwartungen mediiert. Gemäss Hypothese $4 \mathrm{~b}$ erklärten kommunizierte hohe und tiefe Schulniveauerwartungen von Lehrpersonen auch die Leistungsentwicklung in Mathematik vom 5. ins 7. Schuljahr signifikant und substanziell $\left(R^{2}\right.$ change $\left.=8.8 \%\right)$. Wiederum wurden die Schulniveauerwartungen hoch (Bootstrapintervall -.11 bis .12) sowie die Schulniveauerwartungen tief (Bootstrapintervall -.12 bis .11) gemäss Hypothese $5 \mathrm{a}$ nicht durch die leistungsbezogenen Lehrpersonenerwartungen mediiert. 
Tab.5 Kommunizierte Lehrpersonenerwartungen und Schülerleistungen in Mathematik: Gruppe früher Übertritt

\begin{tabular}{|c|c|c|c|c|c|c|}
\hline \multirow[b]{2}{*}{ Modell } & \multicolumn{3}{|c|}{ Leistungen M 6. Sj } & \multicolumn{3}{|c|}{ Leistungen $\mathrm{M}$ 7. Sj } \\
\hline & M1 & M2 & M3 & M1 & M2 & M3 \\
\hline Leistungen M 5. Sj & $.46^{* * *}$ & $.41^{* * *}$ & $.41^{* * *}$ & $.44^{\star * *}$ & $.39^{\star * *}$ & $.39^{* * *}$ \\
\hline IQ $5 . \mathrm{Sj}$ & $.33^{\star * *}$ & $.27^{\star * *}$ & $.27^{\star * *}$ & $.32^{* * *}$ & $.26^{* * *}$ & $.26^{* * *}$ \\
\hline $\begin{array}{l}\text { E: Sozioökonomischer } \\
\text { Status (ISEI) }\end{array}$ & $.25^{\star * *}$ & $.08^{*}$ & .08 & .19 & .04 & .04 \\
\hline Schulni Lp hoch 5. Sj & & .03 & .05 & & $.10^{\star * *}$ & $.11^{\star *}$ \\
\hline Schulni Lp tief 5. Sj & & $-.38^{\star * *}$ & $-.40^{\star * *}$ & & $-.33^{\star * *}$ & $-.33^{* * *}$ \\
\hline L-Erw Lp 5. Sj & & & -.03 & & & -.02 \\
\hline$F, p$ & $158.4^{* * *}$ & $146.3^{\star * *}$ & $121.9^{* * *}$ & $128.6^{\star * *}$ & $110.4^{* * *}$ & $91.8^{* * *}$ \\
\hline$d f_{1} d f_{2}$ & 3,495 & 5,493 & 6,492 & 3,495 & 5,493 & 6,492 \\
\hline$R^{2} a d j$ & $48.7 \%$ & $59.3 \%$ & $59.3 \%$ & $43.5 \%$ & $52.3 \%$ & $52.2 \%$ \\
\hline
\end{tabular}

Legende: M: Mathematik; Schulni Lp: Schulniveauerwartungen Lehrperson; L-Erw Lp: Leistungserwartungen von Lehrpersonen; Sj: Schuljahr; ${ }^{* *}: p<.001 ;{ }^{* *}: p<.01 ;{ }^{*}: p<.05$

Die analogen Regressionsanalysen wurden erneut auch für das Fach Deutsch durchgeführt (Tabelle 6). Hohe und tiefe kommunizierte Schulniveauerwartungen von Lehrpersonen erklärten nach Kontrolle der Intelligenz und des sozioökonomischen Status die Leistungsentwicklung vom 5. zum 6. Schuljahr der Sekundarstufe I signifikant und substanziell $\left(R^{2}\right.$ change $\left.=10.9 \%\right)$. Die Schulniveauerwartungen hoch (Bootstrapintervall -.16 bis .06) und die Schulniveauerwartungen tief (Bootstrapintervall -.07 bis .16) wurden gemäss Hypothese $5 \mathrm{~b}$ nicht durch die leistungsbezogenen Lehrpersonenerwartungen mediiert. Tiefe kommunizierte Schulniveauerwartungen von Lehrpersonen erklärten gemäss Hypothese $4 \mathrm{~b}$ auch die Leistungsentwicklung vom 5. ins 7. Schuljahr der Sekundarstufe I signifikant und substanziell ( $R^{2}$ change $=9.0 \%$ ). Der Effekt wurde gemäss Hypothese $5 \mathrm{~b}$ durch die leistungsbezogenen Lehrpersonenerwartungen nicht mediiert (Bootstrapintervall -.04 bis .25). 
Tab. 6 Kommunizierte Lehrpersonenerwartungen und Schülerleistungen in Deutsch: Gruppe früher Übertritt

\begin{tabular}{|c|c|c|c|c|c|c|}
\hline \multirow[b]{2}{*}{ Modell } & \multicolumn{3}{|c|}{ Leistungen D 6. Sj } & \multicolumn{3}{|c|}{ Leistungen D 7. Sj } \\
\hline & M1 & M2 & M3 & M1 & M2 & M3 \\
\hline Leistungen D 5. Sj & $.42^{* * *}$ & $.34^{* * *}$ & $.35^{\star * *}$ & $.37^{\star * *}$ & $.27^{\star \star \star}$ & $.27^{\star * *}$ \\
\hline IQ $5 . \mathrm{Sj}$ & $.24^{* * *}$ & $.20^{\star * *}$ & $.20^{\star * *}$ & $.19^{\star * *}$ & $.16^{\star * *}$ & $.16^{* * *}$ \\
\hline $\begin{array}{l}\text { E: Sozioökonomischer } \\
\text { Status (ISEI) }\end{array}$ & $.28^{* * *}$ & $.13^{* * *}$ & $.13^{* * *}$ & $.24^{\star * *}$ & $.10^{*}$ & $.10^{* *}$ \\
\hline Schulni Lp hoch $5 . \mathrm{Sj}$ & & $-.06^{\star}$ & -.05 & & .04 & 0.07 \\
\hline Schulni Lp tief 5. Sj & & $-.39^{* * *}$ & $-.41^{\star * *}$ & & $-.36^{\star * *}$ & $-.40^{* * *}$ \\
\hline L-Erw Lp 5. Sj & & & -.04 & & & -0.06 \\
\hline$F, p$ & $148.3^{* * *}$ & $137.8^{\star * *}$ & $114.8^{\star * \star}$ & $88.3^{\star * *}$ & $77.7^{\star * *}$ & $65.0^{* * *}$ \\
\hline$d f_{1} d f_{2}$ & 3,495 & 5,493 & 6,492 & 3,495 & 5,493 & 6,492 \\
\hline$R^{2}$ adj & $47.0 \%$ & $57.9 \%$ & $57.8 \%$ & $34.5 \%$ & $43.5 \%$ & $43.50 \%$ \\
\hline
\end{tabular}

Legende: D: Deutsch; Schulni Lp: Schulniveauerwartungen Lehrperson; L-Erw Lp: Leistungserwartungen von Lehrpersonen; $\mathrm{Sj}$ : Schuljahr; ${ }^{* *}: p<.001 ;{ }^{* *}: p<.01 ;{ }^{*}: p<.05$

Die Ergebnisse waren für Mathematik und Deutsch auf der Konzeptebene nahezu analog. Sie zeigten eine hohe Varianzaufklärung der kommunizierten Schulniveauerwartungen von Lehrpersonen. Überdies werden die kommunizierten Schulniveauerwartungen von Lehrpersonen durch die leistungsbezogenen Lehrpersonenerwartungen nicht mediiert.

\section{Diskussion}

Nicht kommunizierte Schulniveauerwartungen von Lehrpersonen beeinflussen die Leistungsverläufe von Schülerinnen und Schülern in Mathematik und Deutsch in der Primarschule und beim Übergang in die Sekundarstufe I, weil damit unterschiedliche, nicht kommunizierte Leistungserwartungen von Lehrpersonen verbunden sind. Die nicht kommunizierten Schulniveauerwartungen haben auf der Konzeptebene in Mathematik einen stärkeren Effekt als in Deutsch, sowohl bei Verbleib bei der gleichen Klassenlehrperson als auch beim Übertritt in die Sekundarstufe I. Je nach Analyse sind die Effekte der Erwartung auf ein Schulniveau mit hohen bzw. tiefen Ansprüchen aber stärker. Diese Befunde lassen sich gut in die Forschung zu den Erwartungseffekten von Lehrpersonen einordnen (Brophy, 1985; Rubie-Davis et al., 2015) und differenzieren das Konzept der Lehr- 
personenerwartung nach dem Objekt Schulniveau und Leistung. Die Wirkung von nicht kommunizierten Schulniveauerwartungen auf die Leistungen wird durch die leistungsbezogenen Lehrpersonenerwartungen vermittelt, ausser für die Leistungsentwicklung vom 5. zum 7. Schuljahr in Mathematik. Offenbar werden im Fach Mathematik im Unterschied zu Deutsch die leistungsbezogenen Lehrpersonenerwartungen von den Schülerinnen und Schülern nicht verinnerlicht, weshalb sie bei einem Lehrpersonenwechsel keine Wirkungen auf die Leistungen haben. Möglicherweise vergeben Lehrpersonen während des Selektionsverfahrens in Mathematik je nach zugewiesenem Schulniveau unterschiedlich anspruchsvolle Aufgaben, so dass die Erwartungseffekte überlagert werden. Viele Lehrpersonen richten ihren Mathematikunterricht an Aufgaben aus, deren Auswahl an die Fähigkeiten der Schülerinnen und Schüler angepasst wird (Reusser \& Pauli, 2003). Diese Art von adaptivem Unterricht ist im Deutschunterricht womöglich seltener, weshalb sich verinnerlichte leistungsbezogene Erwartungseffekte selbst nach dem Schulübertritt nachweisen lassen.

Neu ist die Unterscheidung zwischen den nicht kommunizierten Schulniveauerwartungen und kommunizierten Schulniveauerwartungen. Sie lassen sich mit zwei unterschiedlichen Prozessen erklären. Während der Effekt der nicht kommunizierten Schulniveauerwartungen durch implizite Leistungserwartungen von Lehrpersonen erklärt wird, konnte dieser Effekt bei kommunizierten Schulniveauerwartungen von Lehrpersonen auf Schülerleistungen nicht gefunden werden. Hier spielen vermutlich geplante, anreichernde Unterrichtsstrategien eine Rolle. Erstmals wird gezeigt, dass sich die Leistungsentwicklung von Schülerinnen und Schülern je nach Schulniveauerwartung bereits vor dem Übergang in die Sekundarstufe I unterscheiden. Die Ergebnisse zeigen in die Richtung, dass bereits beim Eintritt in ein Niveau der Sekundarstufe I ein Schereneffekt der Leistungsentwicklung in Deutsch und Mathematik beobachtet werden kann.

Die kommunizierten Schulniveauerwartungen von Lehrpersonen erklären mehr Varianz der Leistungsveränderung in Deutsch und Mathematik als die nicht kommunizierten Schulniveauerwartungen. Dies ist interessant, weil die Schülerinnen und Schüler, die mit kommunizierten Schulniveauerwartungen konfrontiert worden sind, früher die Lehrperson wechselten als die Schülerinnen und Schüler, an die nicht kommunizierte Schulniveauerwartungen gerichtet werden. Offenbar wirken sich explizite Schulniveauerwartungen, begleitet durch entsprechende didaktische Strategien der Lehrperson und von damit korrespondierendem Fähigkeitsselbstkonzept und Leistungsmotivation des Schülers bzw. der Schülerin, stärker auf die Leistungsentwicklung aus als nicht kommunizierte Schulniveauerwartungen.

Einschränkend muss angemerkt werden, dass aufgrund der fehlenden Mediationseffekte nicht erklärt werden kann, wie kommunizierte Schulniveauerwartun- 
gen konkret auf die Leistungsentwicklung wirken. Es wurden dazu didaktische Erklärungsansätze vorgelegt, die gesondert überprüft werden müssen. Auch wurden allfällige Interaktionen von Lehrpersonen- und Elternerwartungen nicht analysiert. Es wäre sinnvoll, in weiterführenden Analysen Effekte der Lehrpersonenerwartungen auf die Motivation und das Fähigkeitsselbstkonzept zu untersuchen. Diese Fragen können in Folgeanalysen bearbeitet werden. Mit der Differenzierung von verschiedenen Arten von Lehrpersonenerwartungen und mit der Vorhersage der kurz- und mittelfristigen Veränderung von Leistungen vor und nach einem Schulübergang - repliziert in zwei Fächern im Schweizer Schulsystem - wurde illustriert, wie Lehrpersonen wirksam die Leistungsentwicklung von Schülerinnen und Schülern beeinflussen.

\section{Literatur}

Baron, R. M., \& Kenny, D. A. (1986). The moderator-mediator variable distinction in Social Psychological Research: Conceptual, strategic, and statistical considerations. Journal of Personality and Social Psychology, 51, 1173-1182.

Baumert, J., Stanat, P., \& Watermann, R. (2006). Schulstruktur und die Entstehung differenzieller Lern- und Entwicklungsmilieus. In J. Baumert, P. Stanat \& R. Waterman (Hrsg.), Herkunftsbedingte Disparitäten im Bildungswesen: Differenzielle Bildungsprozesse und Probleme der Verteilungsgerechtigkeit (S.95-188). Wiesbaden: VS-Verlag.

Brophy, J. E. (1985). Teacher-student interaction. In J. B. Dusek (Ed.), Teacher expectancies (pp. 303-328). Hillsdale, NJ: Erlbaum.

Ditton, H., \& Krüsken, J. (2009). Bildungslaufbahnen im differenzierten Schulsystem Entwicklungsverläufe von Laufbahnempfehlungen und Bildungsaspirationen in der Grundschulzeit. Zeitschrift für Erziehungswissenschaft, 12(1), 74-102.

Dusek, J. B., \& Joseph, A. (1983). The bases of teacher expectation: A meta-analysis. Journal of Educational Psychology, 75(3), 327-346.

Eccles, J., Adler, T. F., Futterman, R., Goff, S. B., Kaczala, C. M., Meece, J. L., \& Midgley, C. (1983). Expectancies, values, and academic behaviors. In J. T. Spence (Ed.), Achievement and achievement motives (pp. 75-146). San Francisco: Freeman.

Eccles, J. S., \& Roeser, R. W. (2011). Schools as developmental contexts during adolescence. Journal of Research on Adolescence, 21(1), 225-241.

Eccles, J. S., Wigfield, A., \& Schiefele, U. (1998). Motivation to succeed. In W. Damon \& N. Eisenberg (Eds.), Handbook of child psychology: Social, emotional, and personality development (Vol. 3, pp. 1017-1095). New York: Wiley.

Englund, M. M., Egeland, B., \& Collins, W. A. (2008). Exceptions to High School dropout predictions in a low-income sample: Do adults make a difference? Journal of Social Issue, 64(1), 77-94. 
Friedrich, A., Flunger, B., Nagengast, B., Jonkmann, K., \& Trautwein, U. (2015). Pygmalion effects in the classroom: Teacher expectancy effects on students' math achievement. Contemporary Educational Psychology, 41, 1-12. doi:10.1016/j.cedpsych.2014.10.006

Ganzeboom, H. B. G., \& Treiman, D. J. (2010). International stratification and mobility file: Conversion tools. Amsterdam: Department of Social Research Methodology.

Glock, S., Kneer, J., \& Kovacs, C. (2013). Preservice teachers' implicit attitudes toward students with and without immigration background: A pilot study. Studies in Educational Evaluation, 39(4), 204-210. doi:10.1016/j.stueduc.2013.09.003

Haberman, S. J. (2009). Linking parameter estimates derived from an item response model through separate calibrations. Princeton, NJ: Educational Testing Service.

Harris, M. J., \& Rosenthal, R. (1985). Mediation of interpersonal expectancy effects: 31 meta-analyses. Psychological Bulletin, 97, 363-386.

Jussim, L., \& Harber, K. D. (2005). Teacher expectations and self-fulfilling prophecies: Knowns and unknowns, resolved and unresolved controversies. Personality and Psychology Review, 9(2), 131-155.

Jussim, L., Eccles, J., \& Madon, S. (1996). Social perception, social stereotypes, and teacher expectations: Accuracy and the quest for the powerful self-fulfilling prophecy. Advances in Experimental Social Psychology, 28, 281-388.

Jussim, L., Harber, K. D., Crawford, J. T., Cain, T. R., \& Cohen, F. (2005). Social reality makes the social mind: Self-fulfilling prophecy, stereotypes, bias, and accuracy. Interaction Studies, 6(1), 85-102.

Jussim, L., Robustelli, S., \& Cain, T. R. (2009). Teacher expectations and self-fulfilling prophecies. Handbook of motivation at school, 349-380.

Ludwig, P. H. (1998). Pygmalioneffekt. In D. H. Rost (Hrsg.), Handwörterbuch Pädagogische Psychologie (S. 415-419). Weinheim: Beltz.

McKown, C., \& Weinstein, R. S. (2008). Teacher expectations, classroom context, and the achievement gap. Journal of School Psychology, 46(3), 235-261. doi:10.1016/j.jsp.2007.05.001

Moser, U., Buff, A., Angelone, D., \& Hollenweger, J. (2011). Nach sechs Jahren Primarschule. Deutsch, Mathematik und motivational-emotionales Befinden am Ende der 6. Klasse. Zürich: Bildungsdirektion Kanton Zürich.

Neuenschwander, M. P. (2014). Selektionsentscheidungen beim Übergang in die Sekundarstufe I und in den Arbeitsmarkt im Vergleich. In M. P. Neuenschwander (Hrsg.), Selektion in Schule und Arbeitsmarkt (S. 63-98). Zürich: Rüegger.

Neuenschwander, M. P., Rottermann, B., Rösselet, S., \& Niederbacher, E. (2015). Bedingungen von erwartungswidrigen Schulniveauzuweisungen beim Übergang in die Sekundarstufe I. Zeitschrift für Soziologie der Erziehung und Sozialisation, 35(3), 285-303.

Preacher, K. J., \& Hayes, A. F. (2008). Asymptotic and resampling methods for estimating and comparing indirect effects. Behavior Research Methods, 40(3), 879-891.

Reusser, K., \& Pauli, C. (2003). Mathematikunterricht in der Schweiz und in weiteren sechs Ländern (TIMSS-Video Studies). Zürich: Universität Zürich.

Rosenthal, R., \& Jacobson, L. (1974). Pygmalion im Unterricht. Weinheim: Belz.

Rosenthal, R. (1968). Experimenter expectancy and the reassuring nature of the null hypothesis decision procedure. Psychological Bulletin Monograph Supplement, 70(6, part 2), 30-47.

Rubie-Davies, C. M., Peterson, E. R., Sibley, C. G., \& Rosenthal, R. (2015). A teacher expectation intervention: Modelling the practices of high expectation teachers. Contemporary Educational Psychology, 40, 72-85. doi:10.1016/j.cedpsych.2014.03.003 
Tenenbaum, H. R., \& Ruck, M. D. (2007). Are teachers' expectations different for racial minority than for European American students? A meta-analysis. Journal of Educational Psychology, 99(2), 253-273. doi:10.1037/0022-0663.99.2.253.

Trouilloud, D. O., Sarrazin, P. G., Martinek, T. J., \& Guillet, E. (2002). The influence of teacher expectations on student achievement in physical education classes: Pygmalion revisited. European Journal of Social Psychology, 32, 591-607.

Warm, T. A. (1989). Weighted likelihood estimation of ability in item response theory. Psychometrika, 54(3), 427-450.

Weiss, R. H. (2008). CFT 20-R. Grundintelligenztest Skala 2 - Revision - (CFT 20-R) mit Wortschatztest und Zahlenfolgentest - Revision (WS/ZF-R). Göttingen: Hogrefe.

Yen, W. M., \& Fitzpatrick, A. R. (2006). Item response theory. In R. L. Brennan (Ed.), Educational Measurement (pp. 111-154). Westport: Praeger Publisher.

Open Access Dieses Kapitel wird unter der Creative Commons Namensnennung 4.0 International Lizenz (http://creativecommons.org/licenses/by/4.0/deed.de) veröffentlicht, welche die Nutzung, Vervielfältigung, Bearbeitung, Verbreitung und Wiedergabe in jeglichem Medium und Format erlaubt, sofern Sie den/die ursprünglichen Autor(en) und die Quelle ordnungsgemäß nennen, einen Link zur Creative Commons Lizenz beifügen und angeben, ob Änderungen vorgenommen wurden.

Die in diesem Kapitel enthaltenen Bilder und sonstiges Drittmaterial unterliegen ebenfalls der genannten Creative Commons Lizenz, sofern sich aus der Abbildungslegende nichts anderes ergibt. Sofern das betreffende Material nicht unter der genannten Creative Commons Lizenz steht und die betreffende Handlung nicht nach gesetzlichen Vorschriften erlaubt ist, ist für die oben aufgeführten Weiterverwendungen des Materials die Einwilligung des jeweiligen Rechteinhabers einzuholen.

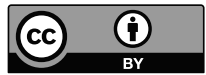

